\title{
Implicit Lyapunov control of finite dimensional Schrödinger equations
}

\author{
Karine Beauchard ${ }^{\mathrm{a}}$, Jean Michel Coron ${ }^{\mathrm{b}}$, Mazyar Mirrahimi ${ }^{\mathrm{c}}$, \\ Pierre Rouchon ${ }^{\mathrm{c}}$, \\ a CMLA, ENS Cachan, avenue du president Wilson, 94230 Cachan, France \\ b Département de Mathématique, Bât. 425, Université Paris-Sud, 91405 Orsay, \\ France \\ ${ }^{\mathrm{c}}$ Centre Automatique et Systèmes, Ecole des Mines de Paris, 60 Bd Saint-Michel, \\ 75272 Paris cedex 06, France
}

\begin{abstract}
An implicit Lyapunov based approach is proposed for generating trajectories of a finite dimensional controlled quantum system. The main difficulty comes from the fact that we consider the degenerate case where the linearized control system around the target state is not controllable. The controlled Lyapunov function is defined by an implicit equation and its existence is shown by a fix point theorem. The convergence analysis is done using LaSalle invariance principle. Closed-loop simulations illustrate the interest of such feedback laws for the open-loop control of a test case considered by chemists.
\end{abstract}

\section{Introduction}

Even if the controllability of a finite dimensional quantum system, $\iota \frac{d}{d t} \Psi=$ $\left(H_{0}+u(t) H_{1}\right) \Psi$ where $\Psi \in \mathbb{C}^{N}$ and $H_{0}$ and $H_{1}$ are $N \times N$ Hermitian matrices with coefficients in $\mathbb{C}$, has been completely explored $[23,19,1,2,25]$, this does not guarantee the simplicity of the trajectory generation. Very often the chemists formulate the task of the open-loop control as a cost functional to be

Email addresses: Karine.Beauchard@math.u-psud.fr (Karine Beauchard), Jean-Michel.Coron@math.u-psud.fr (Jean Michel Coron), mazyar.mirrahimi@polytechnique.org (Mazyar Mirrahimi), pierre.rouchon@ensmp.fr (Pierre Rouchon). 
minimized. Optimal control techniques (see e.g., [14,21]) and iterative stochastic techniques (e.g, genetic algorithms $[7,12]$ ) are then two class of approaches which are most commonly used for this task.

When some non-degeneracy assumptions concerning the linearized system are satisfied, [16] provides another method based on Lyapunov techniques for generating trajectories. The relevance of such a method for the control of chemical models has been studied in [17]. Since measurement and feedback in quantum systems lead to much more complicated models and dynamics than the Schrödinger equation $[9,8,15]$, the stabilization techniques presented in [16] are only used for generating open-loop control laws. Simulating the closedloop system, we obtain a control signal which can be used in open-loop for the physical system. Such kind of strategy has already been applied widely in this framework $[5,13,22,18]$.

In this work as an extension of [16]: we relax the limiting assumptions concerning the controllability of the linearized system around the target state. Let us begin by recalling briefly the main result of [16]. We consider the system

$$
\begin{aligned}
\iota \frac{d}{d t} \Psi & =\left(H_{0}+u(t) H_{1}\right) \Psi, \\
\left.\Psi\right|_{t=0} & =\Psi^{0}, \quad\left\|\Psi_{0}\right\|=1 .
\end{aligned}
$$

As $H_{0}$, the free Hamiltonian, and $H_{1}$, the interaction Hamiltonian, are Hermitian matrices, the state of the system verifies the conservation of probability: $\|\Psi(t)\|=1 \forall t \geq 0$. It thus evolves on the unit sphere of $\mathbb{C}^{N}: \mathbb{S}=\{x \in$ $\left.\mathbb{C}^{N} ;\|x\|=1\right\}$

The idea of [16] consists in considering a control Lyapunov function

$$
V(\Psi)=\frac{1}{2}\|\Psi-\phi\|^{2}
$$

where $H_{0} \phi=\lambda \phi$. In order to simplify the geometry of the state space and consider the physically meaning-less global phase of the state, a fictitious phase control $\omega$ is added to the system:

$$
\iota \frac{d}{d t} \Psi=\left(H_{0}+u H_{1}+\omega\right) \Psi .
$$

The Lyapunov function (2) then leads to a feedback design of the form

$$
u(\Psi)=-a \Im\left(\left\langle H_{1} \Psi \mid \phi\right\rangle\right), \quad \omega(\Psi)=-\lambda-b \Im(\langle\Psi \mid \phi\rangle),
$$

where $a$ and $b$ are positive constants. It is shown in [16] that such a feedback stabilizes almost globally the system if and only if

A the spectrum of $H_{0}$ is not $\lambda$-degenerate and all eigenvectors $\varphi$ of $H_{0}$ different from $\phi$, are coupled directly to $\phi:\left\langle H_{1} \varphi \mid \phi\right\rangle \neq 0$ if $\varphi \neq \phi$. 
Here the spectrum $\left\{\lambda_{\alpha}\right\}_{1 \leq \alpha \leq n}$ of $H_{0}$ is said to be $\lambda$-degenerate when there exist $\alpha$ and $\beta$ in $\{1, \ldots, N\}$ such that $\alpha \neq \beta$ and $\left|\lambda_{\alpha}-\lambda\right|=\left|\lambda_{\beta}-\lambda\right|$.

The assumptions in A correspond to the controllability of the linear tangent system around the target state: $(\phi, u=0, \omega=-\lambda)$. Note that this assumption

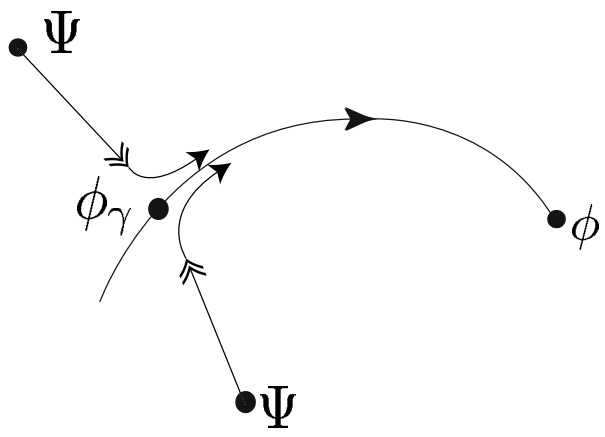

Fig. 1.

may fail to be true for many controllable systems (see the example of section 2). In fact, one might consider any physical system where the direct one-photon transitions between some of the eigenstates do not exist but a multi-photon strategy would ensure the reachability of any eigenstate.

In [16, Section 4] a remedy for such situations is suggested. The method consists in tracking an adiabatic reference trajectory beginning at the target state $\phi$ and ending by a neighborhood of this same state. In this manner we can ensure an approximate control strategy for the systems much more degenerate than the ones considered in assumption $\mathbf{A}$.

The main goal of this paper is to provide another strategy ensuring the almost global stability for such degenerate cases. Let us assume that for any small $\bar{u} \neq 0$ the linear tangent system around $\phi_{\bar{u}}$, the eigenstate of $H_{0}+\bar{u} H_{1}$, is controllable in the sense of $\mathbf{A}$. Then the idea is to introduce a feedback design similar to the last one, replacing the target state $\phi$ by a moving target $\phi_{\gamma(t)}$, where $\gamma(t)$ is defined implicitly by the state of the system $\Psi$. The goal is to make $\phi_{\gamma(t)}$ converging slowly toward $\phi$ and, at the same time, using a feedback design inspired of [16], in order to stabilize as fast as possible the state of the system around the vector function $\phi_{\gamma(t)}$ (see Figure 1). This strategy has been introduced in [6] for the stabilization of the Euler equation of incompressible fluids; in [6], it is $\gamma \nabla \theta$, with $\theta$ as in [6, p.1884, equations (3.47) and (3.48)] which plays the role of $\phi_{\gamma}$ here.

In Section 2, we present the implicit Lyapunov technique for finite dimensional Schrödinger equations. The existence of the implicit control Lyapunov function is ensured by a fix point theorem. Then the convergence of the stabilization technique has been studied. The two cases of an isolated target state and a degenerate one are treated separately. Finally in Section 3, we perform 
some numerical simulations for a 5-dimensional test case borrowed from the quantum chemistry literature. These simulations illustrate the interest of the implicit Lyapunov technique for the open-loop control of the systems considered to be hard by chemists.

\section{Control design}

As a first step (and for some technical reasons), let us change the Lyapunov function introduced in the introduction and let us take

$$
V(\Psi):=1-|\langle\Psi \mid \phi\rangle|^{2}
$$

Thus

$$
\frac{d}{d t} V(\Psi)=-u(t) \Im\left(\left\langle H_{1} \Psi \mid \phi\right\rangle\langle\phi \mid \Psi\rangle\right)
$$

The feedback law

$$
u(\Psi):=c \Im\left(\left\langle H_{1} \Psi \mid \phi\right\rangle\langle\phi \mid \Psi\rangle\right),
$$

with a positive constant $c$ ensures $d V / d t \leq 0$. A convergence analysis analogous to the one proposed in [16] for the feedback law (4) under the additional assumption

$\mathbf{A}_{1}$ : The population of the eigenstate $\phi$ in the initial state (and so in the state of the system at any time $t$ ) is different from zero: $\langle\Psi \mid \phi\rangle \neq 0$;

shows that the convergence of $\Psi$ to the set $\left\{e^{\iota \theta} \phi \mid \theta \in[0,2 \pi)\right\}$ is also equivalent to the controllability of the first variation around the reference trajectory $\left(\Psi_{r}=e^{-\iota \lambda t} \phi, u=0\right)$.

Remark 1 Note that in practice the assumption $\boldsymbol{A}_{1}$ is not really restrictive. In fact if the population of the eigenstate $\phi$ in the initial state is zero it suffices to perturb a little the system at the beginning by using sinusoidal fields with resonant frequencies.

Now let us consider the case where the linear tangent system is not controllable. This might happen for many controllable systems: it corresponds either to a $\lambda$-degenerate spectrum for $H_{0}$ or to the non existence of single-photon transitions in the interaction Hamiltonian between $\phi$ and other eigenstates. The 5-level system [24] corresponding to the internal Hamiltonian $H_{0}$ and the 
dipole moment $H_{1}$ :

$$
H_{0}=\left(\begin{array}{ccccc}
1.0 & 0 & 0 & 0 & 0 \\
0 & 1.2 & 0 & 0 & 0 \\
0 & 0 & 1.3 & 0 & 0 \\
0 & 0 & 0 & 2.0 & 0 \\
0 & 0 & 0 & 0 & 2.15
\end{array}\right) \quad, \quad H_{1}=\left(\begin{array}{lllll}
0 & 0 & 0 & 1 & 1 \\
0 & 0 & 0 & 1 & 1 \\
0 & 0 & 0 & 1 & 1 \\
1 & 1 & 1 & 0 & 0 \\
1 & 1 & 1 & 0 & 0
\end{array}\right)
$$

with $\phi=(1,0,0,0,0)$ provides an example of this kind. This system is controllable: the Lie algebra spanned by $H_{0} / \iota$ and $\mu / \iota$ is $u(5)$ [19]. We will return to this example in the numerical simulations.

For $\gamma \in \mathbb{R}$, let us denote by $\left(\lambda_{n, \gamma}\right)_{1 \leqslant n \leqslant N}$ the eigenvalues of the operator $H_{0}+$ $\gamma H_{1}$, with $\lambda_{1, \gamma} \leqslant \ldots \leqslant \lambda_{N, \gamma}$ and by $\left(\phi_{n, \gamma}\right)_{1 \leqslant n \leqslant N}$ the associated normalized eigenvectors:

$$
\left(H_{0}+\gamma H_{1}\right) \phi_{n, \gamma}=\lambda_{n, \gamma} \phi_{n, \gamma}
$$

In order to simplify the notations, we will take $\phi=\phi_{k}$ for some $k \in\{1,2, . ., N\}$. Then we assume

$\mathbf{A}_{2}$ : There exists a positive constant $\gamma^{*}$ such that, for every $\gamma \in\left(0, \gamma^{*}\right]$, we have $\lambda_{1, \gamma}<\ldots<\lambda_{N, \gamma}$ and the Hamiltonian $H_{0}+\gamma H_{1}$ is not $\lambda_{k, \gamma}$-degenerate. Moreover, for every integer $l \in\{1,2, . ., N\}$ different from $k$, we assume that $\left\langle H_{1} \phi_{k, \gamma} \mid \phi_{l, \gamma}\right\rangle \neq 0$.

The idea is to use the fact that the feedback design presented previously makes the system with internal Hamiltonian $H_{0}+\gamma H_{1}$ and the interaction Hamiltonian $H_{1}$ converging toward $\mathcal{C}_{\gamma}:=\left\{\phi_{k, \gamma} e^{\iota \theta} ; \theta \in \mathbb{R}\right\}$ for every $\gamma \in\left(0, \gamma^{*}\right]$ but not for $\gamma=0$. So we will let $\gamma$ tending to zero and, formally, the convergence toward $\mathcal{C}_{\gamma}$ must be faster than the convergence of $\gamma$ toward 0 (cf. Figure 1 ). There will be two cases to consider:

(1) the case where the target state $\phi_{k}$ is an isolated eigenstate of the free Hamiltonian $H_{0}$ : the dimension of the corresponding eigenspace is 1 ,

(2) the degenerate case.

\subsection{Isolated target state}

We assume that the $k^{\prime}$ th eigenspace of the free Hamiltonian $H_{0}$ is of dimension 1 , so that the target state (i.e. $k$ 'th eigenstate of the system) is defined without 
any ambiguity. We will define a Lyapunov function by

$$
V(\Psi):=1-\left|\left\langle\Psi \mid \phi_{k, \gamma(\Psi)}\right\rangle\right|^{2}
$$

where the function $\Psi \mapsto \gamma(\Psi)$ is implicitly defined as below

$$
\gamma(\Psi):=\theta\left(1-\left|\left\langle\Psi \mid \phi_{k, \gamma(\Psi)}\right\rangle\right|^{2}\right)
$$

for a slowly varying real function $\theta$.

Note that under the assumption of non degeneracy of the $k$ 'th eigenstate of $H_{0}+\gamma H_{1}$ for $\gamma$ in the closed interval $\left[0, \gamma^{*}\right], \phi_{k, \gamma}$ and $\lambda_{k, \gamma}$ are analytic functions of the parameter $\gamma \in\left[0, \gamma^{*}\right]$ [10, Motzkin-Taussky theorem, p85]. In particular, we can consider the derivative of the map $\gamma \mapsto \phi_{k, \gamma}$ at least in the interval $\left[0, \gamma^{*}\right]$. We denote by

$$
\left.\frac{d \phi_{k, \gamma}}{d \gamma}\right|_{\gamma_{0}}
$$

the derivative of this map at the point $\gamma=\gamma_{0}$. Furthermore, as the dependence of $\phi_{k, \gamma}$ with respect to $\gamma$ is analytic, $\frac{d \phi_{k, \gamma}}{d \gamma}$ is bounded on $\left[0, \gamma^{*}\right]$ and thus

$$
C:=\max \left\{\left\|\left.\frac{d \phi_{k, \gamma}}{d \gamma}\right|_{\gamma_{0}}\right\| ; \quad \gamma_{0} \in\left[0, \gamma^{*}\right]\right\}<\infty
$$

A simple computation shows that

$$
\frac{d}{d \gamma} \theta\left(1-\left|\left\langle\Psi \mid \phi_{k, \gamma}\right\rangle\right|^{2}\right)=-2 \theta^{\prime} \Re\left(\left\langle\Psi \mid \frac{d \phi_{k, \gamma}}{d \gamma}\right\rangle\left\langle\phi_{k, \gamma} \mid \Psi\right\rangle\right) .
$$

Taking the function $\theta$ such that $\left\|\theta^{\prime}\right\|_{\infty}$ is small enough (the smallness depending only on $H_{0}, H_{1}, k$ and $\left.\gamma^{*}\right)$ and since $\frac{d \phi_{k, \gamma}}{d \gamma}$ is bounded, the function

$$
\alpha \in\left[0, \gamma^{*}\right] \mapsto \theta\left(1-\left|\left\langle\Psi \mid \phi_{k, \alpha}\right\rangle\right|^{2}\right)
$$

will be contracting for fixed $\Psi \in \mathbb{S}$. Thus, for any fixed $\Psi \in \mathbb{S}$, there exists a unique $\gamma(\Psi) \in\left[0, \gamma^{*}\right]$ such that (9) is verified.

Let us show that $\mathbb{S} \ni \Psi \mapsto \gamma(\Psi) \in\left[0, \gamma^{*}\right]$ defines an application in $C^{\infty}\left(\mathbb{S} ;\left[0, \gamma^{*}\right]\right)$. The implicit function theorem applies here. Let us consider the application

$$
F(\gamma, \psi):=\gamma-\theta\left(1-\left|\left\langle\psi \mid \phi_{k, \gamma}\right\rangle\right|^{2}\right) .
$$

This application $F$ is regular with respect to $\gamma$ and $\psi$ and, for a fixed $\psi \in \mathbb{S}$, we have

$$
F(\gamma(\psi), \psi)=0
$$

Furthermore we have

$$
\frac{d}{d \gamma} F(\gamma, \psi)=1+2 \theta^{\prime} \Re\left(\left\langle\psi \mid \frac{d \phi_{k, \gamma}}{d \gamma}\right\rangle\left\langle\phi_{k, \gamma} \mid \psi\right\rangle\right)
$$


which is non zero for $\theta$ such that $\left\|\theta^{\prime}\right\|_{\infty}$ is small enough. Thus, using the implicit function theorem and the uniqueness of the application $\Psi \mapsto \gamma(\Psi)$, we have the following existence result:

Lemma 2 Let $\theta \in C^{\infty}\left(\mathbb{R}^{+} ;\left[0, \gamma^{*}\right]\right)$ be such that

$$
\theta(0)=0, \theta(s)>0 \text { for every } s>0
$$

$$
\left\|\theta^{\prime}\right\|_{\infty}<\frac{1}{C^{*}} \quad \text { where } \quad C^{*}:=1+\max \left\{\left\|\left.\frac{d \phi_{k, \gamma}}{d \gamma}\right|_{\gamma_{0}}\right\| ; \quad \gamma_{0} \in\left[0, \gamma^{*}\right]\right\} .
$$

Then there exists a unique map $\gamma \in C^{\infty}\left(\mathbb{S} ;\left[0, \gamma^{*}\right]\right)$ such that, for every $\Psi \in \mathbb{S}$,

$$
\gamma(\Psi)=\theta\left(1-\left|\left\langle\Psi \mid \phi_{k, \gamma(\Psi)}\right\rangle\right|^{2}\right) \quad \text { with } \quad \gamma\left(\phi_{k}\right)=0 \text {. }
$$

In the sequel, $\theta \in C^{\infty}\left(\mathbb{R}^{+},\left[0, \gamma^{*}\right]\right)$ is fixed, satisfies (10) and

$$
\left\|\theta^{\prime}\right\|_{\infty} \leqslant 1 / 2 C^{*}
$$

Let $\Psi$ be a solution of the equation

$$
\iota \dot{\Psi}=\left(H_{0}+(\gamma(\Psi)+v(t)) H_{1}\right) \Psi .
$$

We have

$$
\begin{aligned}
\frac{d}{d t} V(\Psi(t))= & -2 v(t) \Im\left(\left\langle H_{1} \Psi(t) \mid \phi_{k, \gamma}\right\rangle\left\langle\phi_{k, \gamma} \mid \Psi(t)\right\rangle\right) \\
& -2 \dot{\gamma}(t) \Re\left(\left\langle\Psi(t)\left|\frac{d \phi_{k, \gamma}}{d \gamma}\right|_{\gamma(t)}\right\rangle\left\langle\phi_{k, \gamma} \mid \Psi(t)\right\rangle\right)
\end{aligned}
$$

A simple computation shows that

$$
\begin{aligned}
\dot{\gamma}(t)=\theta^{\prime}(V) \quad\{ & -2 v(t) \Im\left(\left\langle H_{1} \Psi(t) \mid \phi_{k, \gamma}\right\rangle\left\langle\phi_{k, \gamma} \mid \Psi(t)\right\rangle\right) \\
& \left.-2 \dot{\gamma}(t) \Re\left(\left\langle\Psi(t)\left|\frac{d \phi_{k, \gamma}}{d \gamma}\right|_{\gamma(t)}\right\rangle\left\langle\phi_{k, \gamma} \mid \Psi(t)\right\rangle\right)\right\} .
\end{aligned}
$$

Let us introduce

$$
K(t):=2 \theta^{\prime}(V) \Re\left(\left\langle\Psi(t)\left|\frac{d \phi_{k, \gamma}}{d \gamma}\right|_{\gamma(t)}\right\rangle\left\langle\phi_{k, \gamma} \mid \Psi(t)\right\rangle\right) .
$$

Then, by (12), we have

$$
|K(t)| \leqslant 1 / 2 \text { for every } t \in[0,+\infty)
$$

We have

$$
(1+K(t)) \dot{\gamma}(t)=-2 \theta^{\prime}(V) v(t) \Im\left(\left\langle H_{1} \Psi(t) \mid \phi_{k, \gamma}\right\rangle\left\langle\phi_{k, \gamma} \mid \Psi(t)\right\rangle\right)
$$


and so

$$
\frac{d}{d t} V(\psi(t))=-2 v(t) \Im\left(\left\langle H_{1} \Psi(t) \mid \phi_{k, \gamma}\right\rangle\left\langle\phi_{k, \gamma} \mid \Psi(t)\right\rangle\right)\left\{1+\frac{\theta^{\prime}(V)}{1+K(t)}\right\} .
$$

By (12), we have $\left\|\theta^{\prime}\right\|_{\infty} \leqslant 1 / 2$, which, with (13), gives

$$
1+\frac{\theta^{\prime}(V)}{1+K(t)} \geqslant 0 \quad \text { for every } \quad t \geqslant 0 .
$$

Thus, a feedback law of the form

$$
v(\Psi):=c \Im\left(\left\langle H_{1} \Psi \mid \phi_{k, \gamma(\Psi)}\right\rangle\left\langle\phi_{k, \gamma(\Psi)} \mid \Psi\right\rangle\right)
$$

with a positive constant $c>0$, ensures $d V / d t \leqslant 0$.

The closed-loop system

$$
\iota \dot{\Psi}=\left(H_{0}+(\gamma(\Psi)+v(\Psi)) H_{1}\right) \Psi
$$

admits a global solution in $\mathbb{R}$ since $\Psi \mapsto \gamma(\Psi)$ and $\Psi \mapsto v(\Psi)$ are Lipschitz functions of $\Psi$ and $\|\Psi\|=1$.

A convergence analysis of this feedback design under some suitable assumptions is provided in the next subsection. LaSalle invariance principle will be used in order to characterize the $\omega$-limit set for such a closed-loop system.

\subsection{Convergence analysis}

The main result of this section might be summarized as below

Theorem 3 Let $\theta \in C^{\infty}\left(\mathbb{R}^{+},\left[0, \gamma^{*}\right]\right)$ be such that (10) and (12) hold. Let us also suppose that the assumption $\boldsymbol{A}_{2}$ holds true. Then, the closed-loop system

$$
\iota \frac{d}{d t} \Psi=\left(H_{0}+u(\Psi) H_{1}\right) \Psi, \quad \Psi(0)=\Psi^{0}
$$

with the feedback design

$$
u(\Psi):=\gamma(\Psi)+v(\Psi)
$$

where $\gamma(\Psi)$ is provided by lemma 2 and

$$
v(\Psi):=c \Im\left(\left\langle H_{1} \Psi \mid \phi_{k, \gamma(\Psi)}\right\rangle\left\langle\phi_{k, \gamma(\Psi)} \mid \Psi\right\rangle\right),
$$

where $c$ is a positive constant, admits a global solution on $\mathbb{R}^{+}$. 
Moreover, if $\Psi^{0}$ satisfies

$$
\left\langle\Psi^{0} \mid \phi_{k, \gamma\left(\Psi^{0}\right)}\right\rangle \neq 0
$$

then this solution converges toward $\mathcal{C}:=\left\{\phi_{k} e^{\iota \theta} ; \theta \in \mathbb{R}\right\}$ in the following sense

$$
\lim _{t \rightarrow \infty} \operatorname{dist}(\Psi(t), \mathcal{C})=0
$$

and the set $\left\{\Psi^{0} \in \mathbb{S} ;\left\langle\Psi^{0} \mid \phi_{k, \gamma\left(\Psi^{0}\right)}\right\rangle=0\right\}$ is an unstable invariant manifold for the system (16).

\section{Proof of Theorem 3}

As it is shown in the previous section, $V(\Psi)$ is a control Lyapunov function and, using the feedback design of the Theorem 3 , we have $d V / d t \leq 0$ for every $t \in \mathbb{R}^{+}$.

If $\left\langle\Psi^{0} \mid \phi_{k, \gamma\left(\Psi^{0}\right)}\right\rangle=0$, one can easily see that the second part of the feedback design $v$ will be zero and so $\left\langle\Psi(t) \mid \phi_{k, \gamma(\Psi(t))}\right\rangle=0$, for every $t>0$. Thus the set $\left\{\Psi^{0} \in \mathbb{S} ; \quad\left\langle\Psi^{0} \mid \phi_{k, \gamma\left(\Psi^{0}\right)}\right\rangle=0\right\}$ provides an invariant manifold for the feedback design of Theorem 3 .

Let us assume that $\left\langle\Psi^{0} \mid \phi_{k, \gamma\left(\Psi^{0}\right)}\right\rangle \neq 0$. We use LaSalle invariance principle. Theorem 3.4, page 117 of [11] applies here, so the trajectories of the closedloop system converge to the largest invariant set contained in $d V / d t=0$.

Let us determine this invariant set. Let $\Psi$ be a solution of (16) such that $d V / d t=0$. There exists a constant $\bar{V}$ such that $V(\Psi)=\bar{V}$. This implies that $\gamma(\Psi)$ is constant: $\gamma(\Psi)=\bar{\gamma}$ where $\bar{\gamma}:=\theta(\bar{V})$. The equation $d V / d t=0$ gives

$$
v(\Psi)=\Im\left(\left\langle H_{1} \Psi \mid \phi_{k, \gamma(\Psi)}\right\rangle\left\langle\phi_{k, \gamma(\Psi)} \mid \Psi\right\rangle\right)=0 .
$$

so the function $\Psi$ solves

$$
\iota \frac{d}{d t} \Psi=\left(H_{0}+\bar{\gamma} H_{1}\right) \Psi
$$

There are two cases to consider:

first case: $\bar{\gamma}=0$. We have $\theta(\bar{V})=0$ so, by $(10), \bar{V}=0$ and

$$
\left|\left\langle\Psi \mid \phi_{k}\right\rangle\right|=1
$$

which terminates the proof of the theorem.

second case: $\bar{\gamma} \neq 0$. We have $0<\bar{\gamma}<\gamma^{*}$. Since (18) is verified, we also have

$$
\frac{d}{d t} \Im\left(\left\langle H_{1} \Psi \mid \phi_{k, \gamma(\Psi)}\right\rangle\left\langle\phi_{k, \gamma(\Psi)} \mid \Psi\right\rangle\right)=0
$$


Simple computations give

$$
\begin{aligned}
& \Re\left(\left\langle H_{1}\left(H_{0}+\bar{\gamma} H_{1}\right) \Psi \mid \phi_{k, \bar{\gamma}}\right\rangle\left\langle\phi_{k, \bar{\gamma}} \mid \Psi\right\rangle-\right. \\
& \left.\left\langle H_{1} \Psi \mid \phi_{k, \bar{\gamma}}\right\rangle\left\langle\left(H_{0}+\bar{\gamma} H_{1}\right) \phi_{k, \bar{\gamma}} \mid \Psi\right\rangle\right)=0 .
\end{aligned}
$$

One can easily see that (19) is equivalent to:

$$
\Re\left(\left\langle\left[H_{0}+\bar{\gamma} H_{1}, H_{1}\right] \Psi \mid \phi_{k, \bar{\gamma}}\right\rangle\left\langle\phi_{k, \bar{\gamma}} \mid \Psi\right\rangle\right)=0 .
$$

Similarly, we have

$$
\frac{d}{d t} \Re\left(\left\langle\left[H_{0}+\bar{\gamma} H_{1}, H_{1}\right] \Psi \mid \phi_{k, \bar{\gamma}}\right\rangle\left\langle\phi_{k, \bar{\gamma}} \mid \Psi\right\rangle\right)=0
$$

This implies that

$$
\Im\left(\left\langle\left[H_{0}+\bar{\gamma} H_{1},\left[H_{0}+\bar{\gamma} H_{1}, H_{1}\right]\right] \Psi \mid \phi_{k, \bar{\gamma}}\right\rangle\left\langle\phi_{k, \bar{\gamma}} \mid \Psi\right\rangle\right)=0
$$

Performing similar computations, an easy induction argument shows that

$$
\Re\left(\left\langle a d_{\frac{H_{1}}{\iota}}^{m}\left(\frac{H_{0}+\bar{\gamma} H_{1}}{\iota}\right) \Psi \mid \phi_{k, \bar{\gamma}}\right\rangle\left\langle\phi_{k, \bar{\gamma}} \mid \Psi\right\rangle\right)=0, \quad \forall \quad m \in\{0,1,2, \ldots\} .
$$

Here, $a d_{Z}^{m}(Y)$ is a notation for iterative commutators:

$$
a d_{Z}^{0}(Y)=Z, \quad a d_{Z}^{m}(Y)=\left[Y, a d_{Z}^{m-1}(Y)\right] \quad \text { for } \quad m \geq 1 .
$$

Let us take $\chi=\left\langle\phi_{k, \bar{\gamma}} \mid \Psi\right\rangle \Psi$. We know, by (17), that $\left\langle\phi_{k, \bar{\gamma}} \mid \Psi\right\rangle$ is different from zero at any time $t \geqslant 0$ because it is true for $t=0$. Thus (22) reads

$$
\Re\left(\left\langle a d_{\frac{H_{1}}{\iota}}^{m}\left(\frac{H_{0}+\bar{\gamma} H_{1}}{\iota}\right) \chi \mid \phi_{k, \bar{\gamma}}\right\rangle\right)=0, \quad \forall \quad m \in\{0,1,2, \ldots\} .
$$

By writing the system in the eigenbasis of $H_{0}+\bar{\gamma} H_{1}$, we can assume that $H_{0}+$ $\bar{\gamma} H_{1}$ is diagonal. Then the commutator $\left[H_{0}+\bar{\gamma} H_{1}, B\right]$ where $B=\left(B_{i j}\right)$ is a $N \times$ $N$ matrix might easily be computed. With $H_{0}+\bar{\gamma} H_{1}=\operatorname{diag}\left(\lambda_{1, \bar{\gamma}}, \lambda_{2, \bar{\gamma}}, . ., \lambda_{N, \bar{\gamma}}\right)$, we have

$$
\left[H_{0}+\bar{\gamma} H_{1}, B\right]=\left(\left(\lambda_{i, \bar{\gamma}}-\lambda_{j, \bar{\gamma}}\right) B_{i j}\right)_{i, j} .
$$

Let us take $B=H_{1}$, in order to simplify the notations. We have

$$
a d_{H_{1}}^{m}\left(H_{0}+\bar{\gamma} B\right)=\left(\left(\lambda_{i, \bar{\gamma}}-\lambda_{j, \bar{\gamma}}\right)^{m} B_{i j}\right)_{i, j} .
$$


For sake of simplicity and without loss of generality, let us suppose that $k=1$. Thus the equation (23) reads

$$
\begin{aligned}
\Im\left(\sum_{j} B_{1 j} \chi_{j}\right) & =0, \\
\Re\left(\sum_{j}\left(\lambda_{1, \bar{\gamma}}-\lambda_{j, \bar{\gamma}}\right) B_{1 j} \chi_{j}\right) & =0, \\
\vdots & \\
\Im\left(\sum_{j}\left(\lambda_{1, \bar{\gamma}}-\lambda_{j, \bar{\gamma}}\right)^{2 m} B_{1 j} \chi_{j}\right) & =0, \\
\Re\left(\sum_{j}\left(\lambda_{1, \bar{\gamma}}-\lambda_{j, \bar{\gamma}}\right)^{2 m+1} B_{1 j} \chi_{j}\right) & =0 .
\end{aligned}
$$

Then (25) can be written as $\Im\left(M_{1} \Xi\right)=0$ and $\Re\left(M_{2} \Xi\right)=0$ where

$$
\begin{array}{r}
M_{1}:=\left(\begin{array}{ccc}
1 & \ldots & 1 \\
\left(\lambda_{1, \bar{\gamma}}-\lambda_{2, \bar{\gamma}}\right)^{2} & \ldots & \left(\lambda_{1, \bar{\gamma}}-\lambda_{N, \bar{\gamma}}\right)^{2} \\
\left(\lambda_{1, \bar{\gamma}}-\lambda_{2, \bar{\gamma}}\right)^{4} & \ldots & \left(\lambda_{1, \bar{\gamma}}-\lambda_{N, \bar{\gamma}}\right)^{4} \\
\vdots & \vdots & \vdots \\
\left(\lambda_{1, \bar{\gamma}}-\lambda_{2, \bar{\gamma}}\right)^{2(N-2)} & \ldots & \left(\lambda_{1, \bar{\gamma}}-\lambda_{N, \bar{\gamma}}\right)^{2(N-2)}
\end{array}\right), \\
M_{2}:=\left(\begin{array}{ccc}
\left(\lambda_{1, \bar{\gamma}}-\lambda_{2, \bar{\gamma}}\right) & \ldots & \left(\lambda_{1, \bar{\gamma}}-\lambda_{N, \bar{\gamma}}\right) \\
\left(\lambda_{1, \bar{\gamma}}-\lambda_{2, \bar{\gamma}}\right)^{3} & \ldots & \left(\lambda_{1, \bar{\gamma}}-\lambda_{N, \bar{\gamma}}\right)^{3} \\
\left(\lambda_{1, \bar{\gamma}}-\lambda_{2, \bar{\gamma}}\right)^{5} & \ldots & \left(\lambda_{1, \bar{\gamma}}-\lambda_{N, \bar{\gamma}}\right)^{5} \\
\vdots & \vdots & \vdots \\
\left(\lambda_{1, \bar{\gamma}}-\lambda_{2, \bar{\gamma}}\right)^{2 N-3} & \ldots & \left(\lambda_{1, \bar{\gamma}}-\lambda_{N, \bar{\gamma}}\right)^{2 N-3}
\end{array}\right)
\end{array}
$$

and

$$
\Xi:=\left(B_{12} \chi_{2}, B_{13} \chi_{3}, \ldots, B_{1 N} \chi_{N}\right)^{T} .
$$

Using the Vandermonde criteria and the assumption $\mathbf{A}_{2}\left(\left|\lambda_{1, \bar{\gamma}}-\lambda_{i, \bar{\gamma}}\right| \neq \mid \lambda_{1, \bar{\gamma}}-\right.$ $\lambda_{j, \bar{\gamma}} \mid$ for $\left.i \neq j\right)$, one can see that $M_{1}$ and $M_{2}$ are non-singular real matrices and so $\Im(\Xi)=\Re(\Xi)=0$. Using another time the assumption $\mathbf{A}_{2}\left(B_{1 j} \neq 0\right.$ for $j \neq 1$ ) we deduce that $\chi_{2}=\chi_{3}=\ldots=\chi_{N}=0$ and therefore $\Psi_{2}=\Psi_{3}=\ldots=$ $\Psi_{N}=0$. This implies that $\Psi \in \mathcal{C}$ and finishes the proof of theorem.

\subsection{Degenerate case}

Let us suppose that the eigenspace $E_{k}$ corresponding to the $k$ 'th eigenvalue of the free Hamiltonian $H_{0}$ is of dimension more than one. We will however suppose that, by adding a perturbation of the form $\gamma H_{1}$, for $\gamma \in\left(0, \gamma^{*}\right]$, to 
$H_{0}$, we remove the degeneracy of the matrix. Indeed we suppose that $\mathbf{A}_{2}$ still holds true.

In this case talking about the $k^{\prime}$ th eigenvector as the target state admits an ambiguity: this $k$ 'th eigenvector might be any vector in the eigenspace $E_{k}$ which is not of dimension one here. In order to overcome this ambiguity and also in order to be able to redo the computations of the last section here, we need a result of the perturbation theory for finite dimensional Hermitian operators ([10] page 121):

Lemma 4 Let us consider the $N \times N$ hermitian matrices $H_{0}$ and $H_{1}$ with entries in $\mathbb{C}$ and let us define

$$
H(\gamma):=H_{0}+\gamma H_{1}
$$

For each real $\gamma$, there exists an orthonormal basis $\left(\phi_{n}(\gamma)\right)_{n \in\{1, \ldots, N\}}$ of $\mathbb{C}^{N}$ consisting of eigenvectors of $H(\gamma)$. These orthonormal eigenvectors can be chosen as analytic functions of $\gamma \in \mathbb{R}$.

This lemma shows that the application $\mathbb{R} \ni \gamma \mapsto \phi_{k, \gamma}$ is well defined and is in $C^{\omega}$ and so the decomposition $\gamma \mapsto\left\{\lambda_{1, \gamma}, . ., \lambda_{N, \gamma}\right\}$ and $\gamma \mapsto\left\{\phi_{1, \gamma}, . ., \phi_{N, \gamma}\right\}$ corresponds to the branches of the eigenvalues and eigenvectors depending regularly on $\gamma$.

Using Lemma 4, we can talk about the eigenvectors of the free Hamiltonian without any problem. It suffices to take an eigenvector on a branch of the eigenvectors defined by this lemma. So let us take the eigenvector of $H_{0}$ on the $k$ 'th branch and name it as $\phi_{k}$. The goal is to reach this state by using the approach of the previous subsection. All the computations of the previous subsection might be generalized to this case, because the Lyapunov function $V(\Psi)$ still depends regularly on $\Psi$. Indeed, since $\phi_{k, \gamma}$ is an analytic function of $\gamma$, we can consider the derivative $\frac{d \phi_{k, \gamma}}{d \gamma}$ and, moreover, this derivative is bounded in the interval $\left[0, \gamma^{*}\right]$ which leads to the existence the function $\gamma(\Psi)$ by Lemma 2 . Therefore, Theorem 1 still holds in the degenerate case.

\section{$3 \quad$ Numerical simulations}

Consider the 5-level system defined by (7). We have seen that the linear tangent system around the reference trajectory corresponding to the first eigenstate $\phi_{1}=(1,0,0,0,0)$ of this system is not controllable because the coordinates $\left(H_{1}\right)_{1,2}$ and $\left(H_{1}\right)_{1,3}$ of this matrix are equal to zero. Thus the first Lyapunov approach which consists in using the feedback law

$$
u(t)=c \Im\left(\left\langle H_{1} \Psi \mid \phi_{1}\right\rangle\left\langle\phi_{1} \mid \Psi\right\rangle\right)=c \Im\left(\Psi_{1}^{*}\left(\Psi_{2}+\Psi_{3}\right)\right)
$$



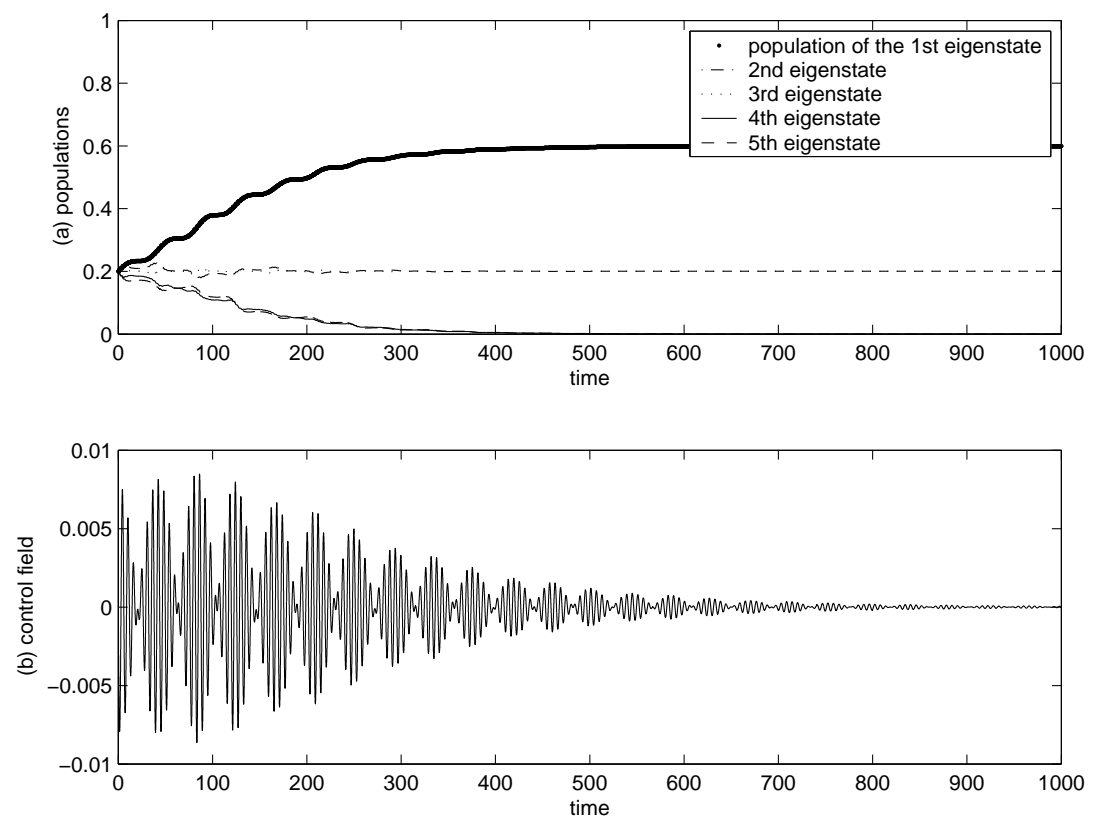

Fig. 2. (a) : the populations of the system trajectory $\Psi(t)$ solution of the system (1) with the feedback design (6). We do not have convergence to $\phi_{1}$ (up to a phase change) as $t \rightarrow+\infty$. (b): The control field found by the feedback design (6).

will not stabilize the system around the reference trajectory corresponding to $\phi_{1}$. Simulations in Figure 2 shows this fact when $c=.02$ and the initial state is $\Psi^{0}=\frac{1}{\sqrt{5}}(1,1,1,1,1)$.

Now let's adapt the feedback design of (15) to this system. The first part of the control field $\gamma(\Psi)$ is defined implicitly by the equation (9). In order to find this function at each time step we use a fixed point algorithm by computing iteratively the value of $\theta\left(V_{\gamma}\right)$. The function $\theta(s)$ is set to be $\theta(s)=\frac{s}{2}$ and the feedback design for the second part is given by (15) where the positive constant $c=.02$ as before. The simulations in Figure 3 show the interest of this approach. Even if $\theta(s)=s / 2$ does not satisfy the condition (11) of Lemma 2, numerical simulations show that the fix point algorithm used in the process of finding $\gamma(\Psi)$ converges. This is due to the fact that the condition (11) is stronger than the condition needed for the fix point scheme.

\section{Conclusion}

A stabilization method for finite dimensional quantum systems has been proposed and its convergence is demonstrated. Even thought the feedback design can not be used for the closed-loop control of a physical system, it can be a useful design tool for generating open-loop trajectories. Moreover the techniques might be useful for more realistic studies concerning the real feedback 

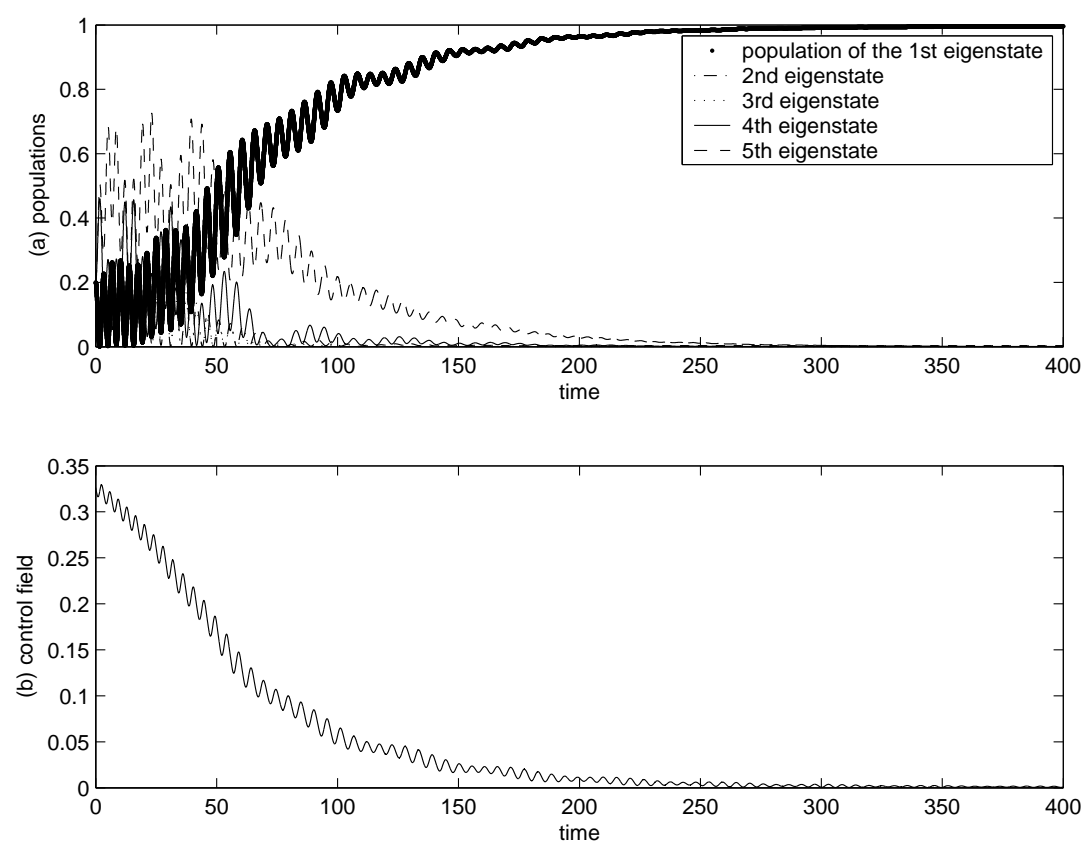

Fig. 3. (a) : the populations of the system trajectory $\Psi(t)$ solution of the system (1) with the feedback design $u(\Psi)=\gamma(\Psi)+v(\Psi)$. As one can easily see the system reaches completely $\phi_{1}$ the first eigenstate of the internal Hamiltonian; (b): the control field $\gamma(\Psi)+v(\Psi)$.

and measurement of the quantum systems [15].

The interest of the method in this paper, in particular, is to overcome the fact that the first order approximation around the reference trajectory is not controllable. Such kind of systems are considered to be hard control problems by the chemists. However, the implicit technique presented in this paper shows relevant for the cases where a small perturbation removes the degeneracy of the system.

A natural question is whether we can extend this kind of results to infinite dimensional systems. A test case to be looked is the control of a quantum particle in a moving potential well. It is proved in [20] that the first order approximation for this system is not controllable and in $[3,4]$ that the nonlinear system is locally controllable around any eigenstate (we are in the same situation as in the 5-level system of the last section). It seems that, for such a system, the assumptions of Theorem 3 are fulfilled. The main problem in dealing with such infinite dimensional systems is the pre-compactness of system trajectories. 


\section{References}

[1] F. Albertini and D. D'Alessandro. Notions of controllability for bilinear multilevel quantum systems. IEEE Transactions on Automatic Control, 48(8):1399-1403, 2003.

[2] C. Altafini. Controllability of quantum mechanical systems by root space decomposition of su(n). Journal of Mathematical Physics, 43(5):2051-2062, 2002 .

[3] K. Beauchard. Local controllability of a 1-D Schrödinger equation. Journal de Mathématique Pures et Appliquées, 84:851-956, 2005.

[4] K. Beauchard and J.M. Coron. Controllability of a quantum particle in a moving potential well. J. Functional Analysis, Accepted, 2005.

[5] Y. Chen, P. Gross, V. Ramakrishna, H. Rabitz, and K. Mease. Competitive tracking of molecular objectives described by quantum mechanics. J. Chem. Phys., 102:8001-8010, 1995.

[6] J.M. Coron. On the null asymptotic stabilization of the two-dimensional incompressible euler equations in a simply connected domain. SIAM J. Control Optim., 37:1874-1896, 1999.

[7] J. M. Geremia and H. Rabitz. Optimal hamiltonian identification: The synthesis of quantum optimal control and quantum inversion. J. Chem. Phys, 118(12):5369-5382, 2003.

[8] R. Van Handel, J. K. Stockton, and H. Mabuchi. Feedback control of quantum state reduction. IEEE Trans. Automat. Control, 50:768-780, 2005.

[9] S. Haroche. Contrôle de la décohérence: théorie et expériences, 2004. Notes de cours, Collège de France. http://www.lkb.ens.fr/recherche/qedcav/college/college.html.

[10] T. Kato. Perturbation Theory for Linear Operators. Springer, 1966.

[11] H.K. Khalil. Nonlinear Systems. MacMillan, 1992.

[12] B. Li, G. Turinici, V. Ramakrishna, and H. Rabitz. Optimal dynamic discrimination of similar molecules through quantum learning control. J. Phys. Chem. B., 106(33):8125-8131, 2002.

[13] Z.-M. Lu and H. Rabitz. Unified formulation for control and inversion of molecular dynamics. J. Phys. Chem., 99:13731-13735, 1995.

[14] Y. Maday and G. Turinici. New formulations of monotonically convergent quantum control algorithms. J. Chem. Phys, 118(18), 2003.

[15] M. Mirrahimi and R. Van Handel. Stabilizing feedback controls for quantum systems. 2005. Submitted, http://arxiv.org/abs/math-ph/0510066. 
[16] M. Mirrahimi, P. Rouchon, and G. Turinici. Lyapunov control of bilinear Schrödinger equations. Automatica, 41:1987-1994, 2005.

[17] M. Mirrahimi, G. Turinici, and P. Rouchon. Reference trajectory tracking for locally designed coherent quantum controls. J. of Physical Chemistry A, 109:2631-2637, 2005.

[18] H. Rabitz and W. Zhu. Quantum control design via adaptive tracking. J. Chem. Phys., 119(7), 2003.

[19] V. Ramakrishna, M. Salapaka, M. Dahleh, and H. Rabitz. Controllability of molecular systems. Phys. Rev. A, 51(2):960-966, 1995.

[20] P. Rouchon. Control of a quantum particle in a moving potential well. In IFAC 2nd Workshop on Lagrangian and Hamiltonian Methods for Nonlinear Control, April 2003.

[21] S. Shi, A. Woody, and H. Rabitz. Optimal control of selective vibrational excitation in harmonic linear chain molecules. J. Chem. Phys., 88(11):6870$6883,1988$.

[22] M. Sugawara. General formulation of locally designed coherent control theory for quantum systems. J. Chem. Phys., 118(15):6784-6800, 2003.

[23] H.J. Sussmann and V. Jurdjevic. Controllability of nonlinear systems. J. Differential Equations, 12:95-116, 1972.

[24] G. Turinici and H. Rabitz. Quantum wavefunction controllability. Chem. Phys., $267: 1-9,2001$.

[25] G. Turinici and H. Rabitz. Wavefunction controllability in quantum systems. J. Phys. A, 36:2565-2576, 2003. 